\title{
Macroevolutionary and morphofunctional patterns in theropod skulls: A morphometric approach
}

Christian Foth and Oliver W.M. Rauhut

Acta Palaeontologica Polonica 58 (1), 2013: 1-16 doi: http://dx.doi.org/10.4202/app.2011.0145

Theropod dinosaurs are one of the most remarkable lineages of terrestrial vertebrates in the Mesozoic, showing high taxonomic and ecological diversity. We investigate the cranial diversity of non-avian theropods and some basal birds, using geometric morphometrics to obtain insights into the evolutionary modifications of the skull. Theropod skulls mostly vary in the shape of the snout and length of the postorbital region (principal component [PC] 1), with further variation in orbit shape, depth of the postorbital region, and position of the jaw joint (PC 2 and PC 3). These results indicate that the cranial shape of theropods is closely correlated with phylogeny and dietary preference. Skull shapes of non-carnivorous taxa differ significantly from carnivorous taxa, suggesting that dietary preference affects skull shape. Furthermore, we found a significant correlation between the first three PC axes and functional proxies (average maximum stress and an indicator of skull strength). Interestingly, basal birds occupy a large area within the morphospace, indicating a high cranial, and thus also ecological, diversity. However, we could include only a small number of basal avialan species, because their skulls are fragile and there are few good skull reconstructions. Taking the known diversity of basal birds from the Jehol biota into account, the present result might even underestimate the morphological diversity of basal avialans.

Key words: Theropoda, geometric morphometrics, macroevolution, feeding ecology, biomechanics, avian evolution, Mesozoic.

Christian Foth [christian.foth@gmx.net], Bavarian State Collection for Palaeontogy and Geology, Department of Earth and Environmental Sciences, Ludwig-Maximilians-University, Richard-Wagner-Str. 10, D-80333 Munich, Germany; Germany; Oliver W.M. Rauhut [o.rauhut@1rz.uni-muenchen.de], Bavarian State Collection for Palaeontogy and Geology, Department of Earth and Environmental Sciences, Ludwig-Maximilians-University, and GeoBioCenter, Ludwig-Maximilians-University, Richard-Wagner-Str. 10, D-80333 Munich, Germany. 
This is an open-access article distributed under the terms of the Creative Commons

Attribution License (for details please see creativecommons.org), which permits unrestricted use, distribution, and reproduction in any medium, provided the original author and source are credited.

\author{
For Full text $(467.1 \mathrm{kB})$ । \\ FoF Supplementary file $(5,058.0 \mathrm{kB})$
}

